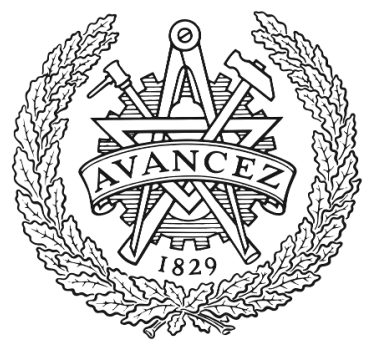

CHALMERS

UNIVERSITY OF TECHNOLOGY

\title{
Multi-color emission with orthogonal input triggers from a diarylethene pyrene-OTHO organogelator cocktail
}

Downloaded from: https://research.chalmers.se, 2023-04-26 12:49 UTC

Citation for the original published paper (version of record):

Johnstone, M., Hsu, C., Hochbaum, N. et al (2020). Multi-color emission with orthogonal input triggers from a diarylethene pyrene-OTHO

organogelator cocktail. Chemical Communications, 56(6): 988-991.

http://dx.doi.org/10.1039/c9cc08994b

N.B. When citing this work, cite the original published paper. 


\section{Check for updates}

Cite this: Chem. Commun., 2020, 56, 988

Received 18th November 2019 Accepted 13th December 2019

DOI: $10.1039 / c 9 c c 08994 b$

rsc.li/chemcomm

\section{Multi-color emission with orthogonal input triggers from a diarylethene pyrene-OTHO organogelator cocktail $\dagger$}

\author{
Mark D. Johnstone, (D) Chien-Wei Hsu, (D) $\ddagger$ Nicolas Hochbaum, $\$$ \\ Joakim Andréasson (D) * and Henrik Sundén (D)*
}

\begin{abstract}
We report on a pyrene-decorated supramolecular gelator based on an oxotriphenylhexanoate (OTHO) that can switch emission profiles between the solution and gel phase. A cocktail of the gelator and a photochromic diarylethene derivative enables four distinct emissive states to be obtained, which are modulated with light and heat as orthogonal input triggers.
\end{abstract}

Supramolecular gels ${ }^{1}$ resulting from self-assembly of lowmolecular-weight gelators (LMWGs) present a robust medium for reversible sol-gel transformations that can be controlled by external stimuli, such as heat, ultrasound, ions and light. ${ }^{2}$ Introducing luminescent chromophores to LMWGs enables physical transformations to be displayed as optical signals that can be easily recognized by the naked eye. ${ }^{3}$ Phenomena such as aggregation induced emission (AIE), ${ }^{4}$ and excimer formation, ${ }^{5}$ are commonly employed as a means of modulating emission intensity or color in input responsive systems. Additionally, chromophores that give optical responses to changes in their aggregation state or intermolecular stacking simultaneously provide in situ information on the molecular packing of the gels, complementary to infrared and NMR spectroscopic techniques. ${ }^{6}$

Combining chromophores with orthogonal switching mechanisms, together with optically responsive LMWGs gels, is crucial for accessing a wider range of colored states. Light is a highly appealing input stimuli as the intensity and wavelength can be easily tuned, in combination with the unsurpassed spatiotemporal control (where and when to apply the stimulus). Accordingly, photochromic molecules have received widespread attention due to their robust reversible photoinduced isomerization reactions between the two, spectrally well-resolved, isomers. ${ }^{7}$ Photoswitches such as diarylethenes (DAEs) have been used extensively for generating multiple distinct colored states in

Chalmers University of Technology, Department of Chemistry and Chemical Engineering, Kemivägen 10, 412 96, Göteborg, Sweden.

E-mail: sundenh@chalmers.se, a-son@chalmers.se

$\dagger$ Electronic supplementary information (ESI) available. See DOI: 10.1039/c9cc08994b

¥ Chien-Wei Hsu and Nicolas Hochbaum contributed equally to the paper. photochromic materials using absorption as the information readout. ${ }^{8}$ However, the low sensitivity of UV/Vis absorption measurements, and the necessity for external circuitry to determine the readout value $\left(A=\log I_{0} / I\right)$ limits the utility of this approach. ${ }^{9}$ In comparison, fluorescence mode detection offers much higher sensitivity, ${ }^{10}$ making emissive systems that can switch among multiple emissive states much more attractive. Although tunable multicolored emission systems have been reported involving a variety of systems such as nanoparticles, ${ }^{11}$ micelles, ${ }^{12}$ quantum dots, ${ }^{13}$ thin films, ${ }^{14}$ and gels, ${ }^{15}$ these typically involve a maximum of three emissive states. To advance this research field, systems capable of generating four or more distinct emissive states must be developed, preferably where the switching is achieved via true orthogonal inputs. Notably, emissive four-state systems have not been reported in multistimuli-responsive materials using photoswitching in conjunction with thermoresponsive gels.

Recently we reported supramolecular systems based on micelles ${ }^{16}$ and LMWGs,${ }^{17}$ in combination with mixed fluorophore systems (referred to as "cocktails") that were capable of multi-color emission via all-photonic modulation. Such systems involve strategic utilization of energy transfer between chromophores in cocktails consisting of DAEs and fluorescent donor molecules.

Herein, we report the synthesis, and the rheological and photophysical characterization of a pyrene-functionalized oxotriphenylhexanoate (ОTHO) gelator and its utilization in a stimuli-responsive multi-color fluorescence system. A modular synthetic approach to the OTHO enables a fluorescent unit to be readily incorporated into the gelator, which in combination with a fluorescent photoswitch produces a multicolor emission system with orthogonal inputs (Fig. 1). The pyrene-decorated OTHO gelator shows substantial differences between the sol and gel state emission color, while the addition of a fluorescent DAE photoswitch enables a larger range of emission colors to be produced. Controlling the sol-gel transformation with heat and the isomeric state of the DAE derivative with light, $4\left(2^{2}\right)$ distinct emissive states can be reached using two external stimuli. 


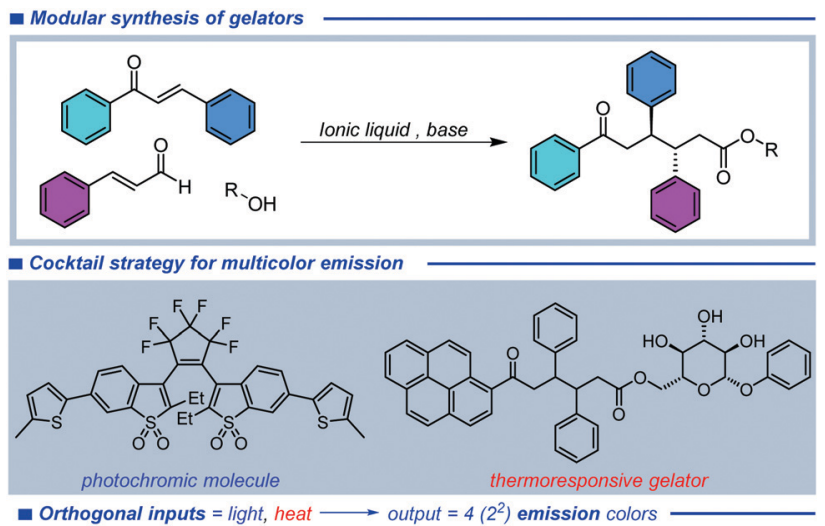

Fig. 1 Design of organogelator with fluorescent pyrene unit and the cocktail strategy for stimuli-responsive multi-color emission.

The input triggers are highly orthogonal as the emission from the DAE photoswitch does not change upon gelation or heat exposure and likewise light exposure (for DAE isomerization) does not influence the intrinsic spectral properties of the pyrene fluorophore.

The chemical structure of the pyrene-based OTHO gelator and photoswitch DAE8 are shown in Scheme 1. DAE8 was synthesized according to the literature procedure. ${ }^{18}$ The OTHO gelator was synthesized in an ionic liquid-mediated threecomponent reaction ${ }^{19}$ where the pyrene-containing chalcone, cinnamaldehyde, and phenyl- $\beta$-D-glucopyranoside were mixed in the presence of EMIMAc and DBU at room temperature. Following flash chromatography, OTHO5 was isolated in $66 \%$ yield (see ESI $\dagger$ for full characterization data including ${ }^{1} \mathrm{H},{ }^{13} \mathrm{C}$ NMR spectra, and HR-MS). The gelating ability of OTHO5 was investigated using the inverted vial method with a range of solvents and solvent mixtures. OTHO5 gelates in toluene, dichlorobenzene, ethyl acetate and in mixtures of toluene and acetonitrile (Table S1 and Fig. S1, ESI $\dagger$ ). The minimum gelation concentration of OTHO5 in toluene was $9 \mathrm{mg} \mathrm{mL} \mathrm{mL}^{-1}$.

Dynamic shear oscillation experiments were performed to evaluate the rheological properties of the gel. The gel displays typical viscoelastic behavior of a physical gel in the frequency sweep (Fig. 2A), where the moduli are largely frequency
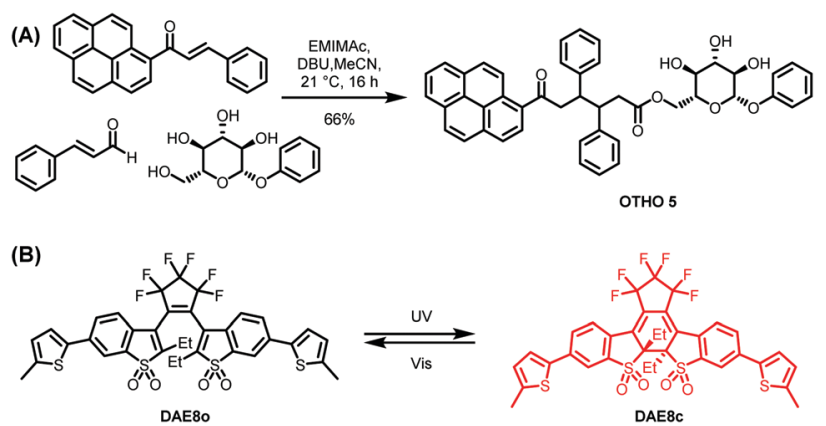

Scheme 1 (A) Three-component synthesis of the OTHO5 gelator (B) Chemical structure of the open (DAE80) and closed (DAE8C) isomeric form of the photoswitch.

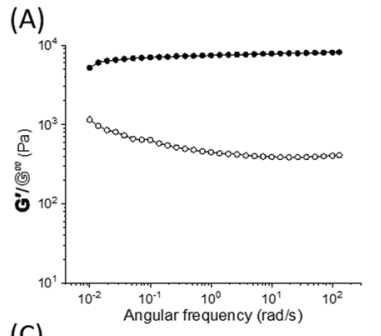

(B)

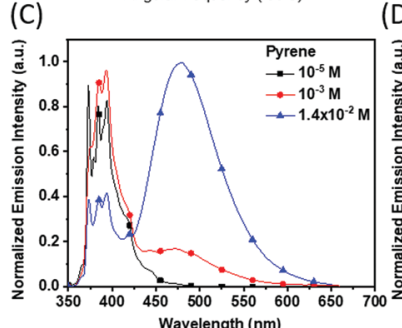

(D)
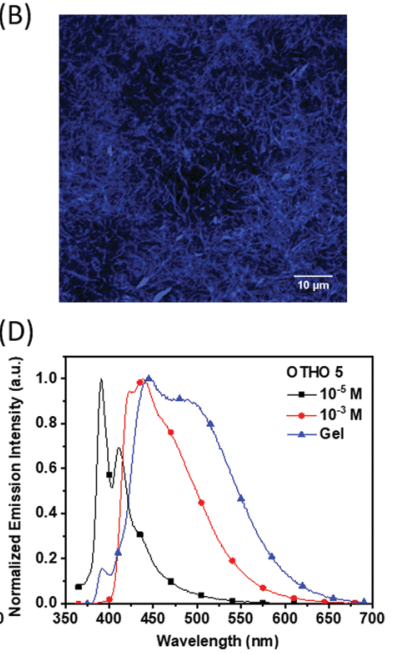

Fig. 2 (A) Frequency sweep of OTHO5 gel in toluene $10 \mathrm{mg} \mathrm{mL}^{-1}$, storage modulus ( ) and loss modulus (O), $0.5 \%$ strain, $20^{\circ} \mathrm{C}$. (B) Confocal laser scanning microscopy image of OTHO5 gel, $405 \mathrm{~nm}$ laser excitation. (C) Emission spectra of pyrene in toluene solution, $\lambda_{\mathrm{ex}}=340 \mathrm{~nm}$. (D) Emission spectra of OTHO5 in toluene solution and gel, $\lambda_{\mathrm{ex}}=370 \mathrm{~nm}$.

independent and the storage modulus $\left(G^{\prime}\right)$ is approximately one order of magnitude larger than the loss modulus $\left(G^{\prime \prime}\right)$. These properties are characteristic for LMWG and other supramolecular entangled fibrillar systems. ${ }^{1 a, 20}$ Due to the strongly fluorescent nature of the pyrene gel, confocal laser scanning microscopy (CLSM) was used to gain insight into the structure of the gel. The CLSM images confirm the presence of a highly entangled fibrillar network in the gel, with the diameter of the fibers in the order of $1 \mu \mathrm{m}$ (Fig. 2B).

The fluorescent properties of OTHO5 were investigated in solution and in the gel phase (Fig. 2). In toluene at low concentrations $\left(10^{-5} \mathrm{M}\right)$, OTHO5 exhibits monomer-like pyrene emission (Fig. 2C and D) with a quantum yield of 0.26. At higher concentrations $\left(>10^{-3} \mathrm{M}\right)$, OTHO5 begins to aggregate and assembly into fibrils, where a moderate component belonging to the excimer emission is present. In the gel phase, OTHO5 shows a broad emission band with mixed components from excimers and other restricted conformations present in the gel. The fluorescence lifetimes of OTHO5 gel in toluene were determined by time-correlated single photon counting. The lifetimes of OTHO5 monomer and excimer were substantially shorter than those of pyrene solutions (see Fig. S8 and S9, ESI $\dagger$ ). ${ }^{21}$ Interestingly, the risetime observed with pyrene solution excimer emission is not present in the gel, indicating that excimer formation is no longer a dynamic process and the pyrene units are confined in the rigid environment of the gel fibers. ${ }^{22}$

Gelation of OTHO5 occurs rapidly in toluene upon cooling to room temperature. In acetonitrile, however, it remains in solution or in a partial gel state. In the case of toluene and acetonitrile mixtures, there are no observable changes in gelation at higher toluene/acetonitrile ratio (9:1), but with increasing acetonitrile, the gel forms slowly upon cooling, while sonication accelerates the gelation process and improves sample uniformity. When the acetonitrile ratio is more than $50 \%$, 

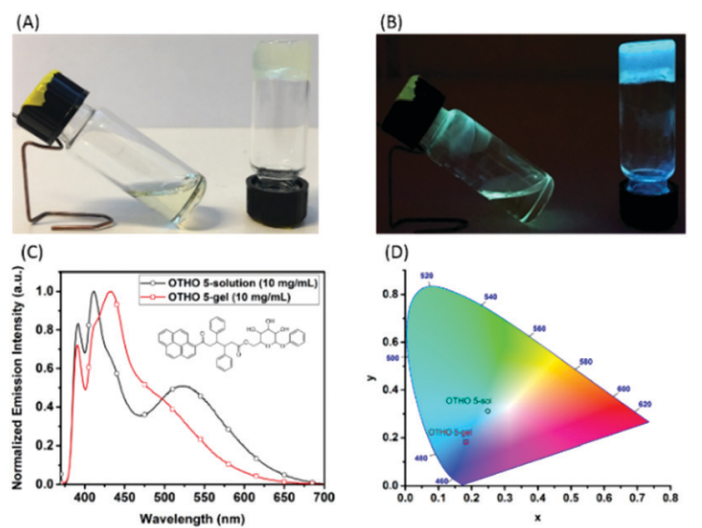

Fig. 3 (A) Photos of OTHO5 gel under ambient light. Left: After heating (sol). Right: Before heating (gel). (B) Photos of OTHO5 gel under $365 \mathrm{~nm}$ light. Left: After heating (sol). Right: Before heating (gel). (C) Emission spectra of OTHO5 in solution and gel, $\lambda_{\text {ex }}=370 \mathrm{~nm}$. (D) CIE diagram of OTHO5 in solution and gel. The concentration of OTHO5 is $10 \mathrm{mg} \mathrm{mL}^{-1}$, toluene/acetonitrile $=2: 1$.

OTHO5 does not fully gelate, but instead dissolves or forms a partial gel even after sonication. Fig. 3A shows OTHO5 $\left(10 \mathrm{mg} \mathrm{mL}^{-1}\right)$ in the $2: 1$ ratio of toluene/acetonitrile. Here, it remains temporarily in solution at room temperature (left) but will gelate immediately when sonication is applied (right). Under UV light irradiation the emission color is dramatically different in solution and in the gel (Fig. 3B). It can be clearly seen that OTHO5 in the sol-state presents excimer emission centered at $525 \mathrm{~nm}$ (Fig. 3C). A tentative explanation is that this is an effect of the more polar solvent mixture that stabilizes the excimer in the sol-state as compared to toluene (Fig. 2D). However, when OTHO5 forms a gel, the excimer emission intensity is less pronounced. This observation is likely due to self-assembly into initial 1D fibrillar structures and a subsequent breakage of excimer formation between the pyrene molecules. ${ }^{5 a, 23}$ Thus, the excimer emission is much more pronounced in solution compared to the gel state. Mapping these emission spectra in the CIE diagram, the coordinates for the solution and the gel are clearly distinct (Fig. 3D).

Having found optimal conditions for a heat-controlled sol-gel transition, corresponding to two signals for optical read-out, we opted for a system containing an additional fluorophore with tunable spectral properties. We were particularly interested in devising a bi-component system where the input triggers for the two fluorophores are orthogonal, implying that the respective fluorophore could be addressed with a high degree of selectivity. Accordingly, OTHO5 gelator $\left(10 \mathrm{mg} \mathrm{mL}^{-1}, 1.4 \times 10^{-2} \mathrm{M}\right)$ and DAE8 $\left(10^{-4} \mathrm{M}\right)$ were dissolved in toluene/acetonitrile $(2: 1)$. By defining the two input triggers as heat (controlling the sol-gel transformation, affecting only the pyrene emission in OTHO5) and UV exposure (isomerizing DAE8 quantitatively to the closed fluorescent isomeric form without affecting the intrinsic pyrene emission in OTHO5) there will be $4\left(2^{2}\right)$ distinct states generated. These are OTHO5-Sol/DAE80, OTHO5Gel/DAE8o, OTHO5-Sol/DAE8c, and OTHO5-Gel/DAE8c. Fig. 4A shows the overall emission spectra of OTHO5/DAE8 in these four situations. With the DAE in the open form (no UV exposure),
(A)
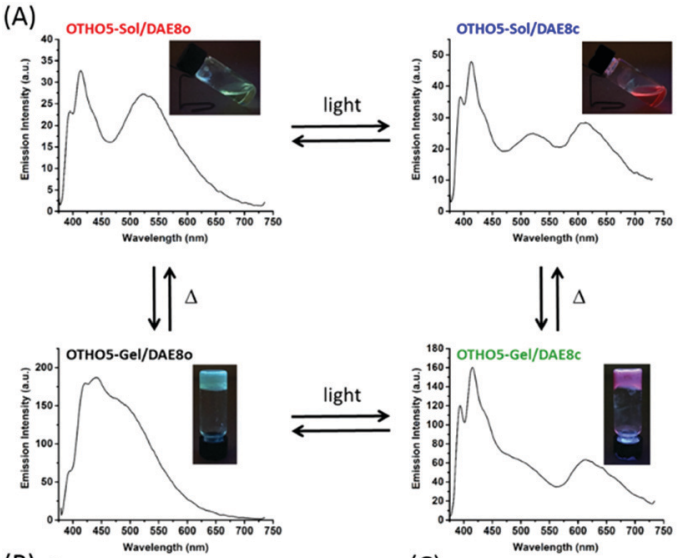

(B)

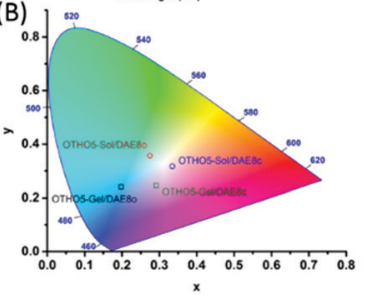

(C)
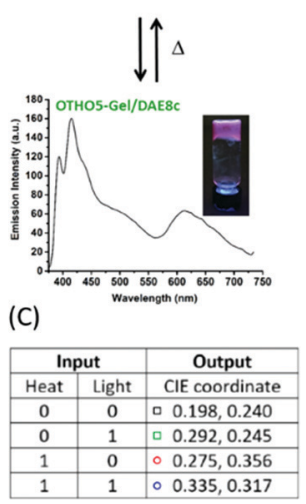

Fig. 4 (A) Multicolored emission triggered by solution-gel transformation (heat) and UV irradiation for isomerization of DAE80 to DAE8C $(\lambda=380 \mathrm{~nm})$. (B) Mapping the CIE coordinates of the four emission spectra in the CIE diagram. (C) CIE coordinates of OTHO5/DAE8 with different combinations of the input triggers. The concentration of OTHO5 is $10 \mathrm{mg} \mathrm{mL}^{-1}$, DAE8 is $10^{-4} \mathrm{M}$, toluene/acetonitrile $=2: 1$. The spectra were recorded at room temperature with $\lambda_{\text {ex }}=370 \mathrm{~nm}$.

the OTHO5/DAE80 mixture exhibits blue emission in gel state and green emission in solution state. However, when exposed to UV light DAE8 isomerizes quantitatively from the open form to the closed form and emits in the red region (see Fig. S4, ESI $\dagger$ for the emission spectrum of DAE8c). Thus, OTHO5/DAE8c displays pink emission in gel state and orange emission in solution state. Although energy transfer from pyrene to DAE8 is also contributing to the spectral changes, the mechanism is mainly "trivial": emission from pyrene is followed by reabsorption of DAE8. FRET is not efficient here due to the low concentration of DAE8 $\left(R_{\text {ave }} \gg R_{0}\right.$, see ESI $\left.\dagger\right)$. Mapping the four emission profiles into the CIE diagram (Fig. 4B) clearly shows the four distinct emissive states that can be achieved with these input triggers (heat and UV-light). It is interesting to note that virtually perfect white light is being generated upon isomerizing the DAE photoswitch between the open and the closed isomer in the sol-state (actual CIE coordinate $(0.33,0.32)$ compared to $(0.33,0.33)$ for perfect white light). As for the concentration of DAE8, there is a tradeoff between the emission intensity from pyrene in OTHO5/DAE8o (high concentration of DAE8 results in a too low intensity) and the emission intensity from DAEc in OTHO5/DAE8c (low concentration of DAE8 results in a too low intensity). A concentration of $10^{-4} \mathrm{M}$ was optimal with respect to generating four distinct CIE coordinates with maximal separation to yield a large spread in the observed emission colors. Fig. S6B (ESI $\dagger$ ) exemplifies the response of the system to differences in the concentration of DAE8. 
To test whether the emission from DAE8c was affected by switching between the solution and gel state, the emission spectra were recorded in the presence of an OTHO gelator lacking the pyrene unit (OTHO3). The absorption and emission profiles were unchanged between solution and gel state (Fig. S5 and S6A, ESI $\dagger$ emission CIE coordinates: 0.644, 0.356, $\Phi_{\mathrm{f}}=0.25$ ), demonstrating that the multi-colored emission system employs truly orthogonal input triggers.

In summary, a pyrene-modified OTHO that gelates in a range of solvents has been devised. It manifests different degrees of pyrene monomer and excimer emission in solution and gel state owing to the self-assembly and disruption of the excimer formation. Furthermore, a multi-color readout system has been realized by combining the photophysical/spectral changes that accompany the sol-gel transformation with a fluorescent DAE photoswitch. The use of two highly orthogonal inputs, heat and light, enables the bi-component OTHO/DAE cocktail to generate four distinct emissive states. This is a powerful approach to multi-wavelength readout systems, offering a situation where substantially more information can be encoded, compared to the binary on-off situations displayed by systems built on single fluorophore emission. Increasing the number of inputs to three stimuli could potentially generate $8\left(2^{3}\right)$ distinct output states, ${ }^{24}$ although this becomes increasingly challenging with mixed chromophore systems if orthogonal switching is desired. Moreover, accessing a larger region of the CIE space would undoubtedly add to the appeal, and could imply the inclusion of a green emitter. Efforts along these lines are presently being undertaken in our laboratory.

We gratefully acknowledge the Swedish Research council (VR and Formas) for funding and Carl-Tryggers foundation and the Swedish Energy Agency for providing postdoc fellowships for C.-W. H. and M. D. J., respectively. Chalmers Excellence Initiative in Nano-science and Nanotechnology, Adlerbert Research Foundation and Wilhelm and Martina Lundgren Research Foundation are also acknowledged for funding.

\section{Conflicts of interest}

There are no conflicts to declare.

\section{References}

1 (a) P. Terech and R. G. Weiss, Chem. Rev., 1997, 97, 3133; (b) J. W. Steed, Chem. Commun., 2011, 47, 1379.

2 (a) C. D. Jones and J. W. Steed, Chem. Soc. Rev., 2016, 45, 6546; (b) E. R. Draper and D. J. Adams, Chem. Commun., 2016, 52, 8196; (c) Z. Sun, Q. Huang, T. He, Z. Li, Y. Zhang and L. Yi, ChemPhysChem, 2014, 15, 2421; (d) S. Yagai and A. Kitamura, Chem. Soc. Rev., 2008, 37, 1520; (e) Z. Ding, Y. Ma, H. Shang, H. Zhang and S. Jiang, Chem. - Eur. J., 2019, 25, 315; $(f)$ X. Wang, Z. Ding, Y. Ma, Y. Zhang, H. Shang and S. Jiang, Soft Matter, 2019, 15, 1658; (g) X. Cao, Y. Li, Y. Yu, S. Fu, A. Gao and X. Chang, Nanoscale, 2019, 11, 10911; (h) J. W. Steed, Chem. Soc. Rev., 2010, 39, 3686; (i) M.-O. M. Piepenbrock, G. O. Lloyd, N. Clarke and J. W. Steed, Chem. Rev., 2010, 110, 1960.

3 (a) S. S. Babu, V. K. Praveen and A. Ajayaghosh, Chem. Rev., 2014, 114, 1973; (b) S. Wang, W. Shen, Y. Feng and H. Tian, Chem. Commun., 2006, 1497; (c) S. Xiao, Y. Zou, M. Yu, T. Yi, Y. Zhou, F. Li and C. Huang, Chem. Commun., 2007, 4758.
4 (a) B.-K. An, D.-S. Lee, J.-S. Lee, Y.-S. Park, H.-S. Song and S. Y. Park, J. Am. Chem. Soc., 2004, 126, 10232; (b) B.-K. An, J. Gierschner and S. Y. Park, Acc. Chem. Res., 2012, 45, 544; (c) Z. Zhao, J. W. Y. Lam and B. Z. Tang, Soft Matter, 2013, 9, 4564; (d) Y. Hong, J. W. Y. Lam and B. Z. Tang, Chem. Soc. Rev., 2011, 40, 5361.

5 (a) Y. Kamikawa and T. Kato, Langmuir, 2007, 23, 274; (b) C. Dou, D. Chen, J. Iqbal, Y. Yuan, H. Zhang and Y. Wang, Langmuir, 2011, 27, 6323; (c) N. Yan, Z. Xu, K. K. Diehn, S. R. Raghavan, Y. Fang and R. G. Weiss, Langmuir, 2013, 29, 793; (d) C.-B. Huang, L.-J. Chen, J. Huang and L. Xu, RSC Adv., 2014, 4, 19538.

6 (a) T. Shu, J. Wu, M. Lu, L. Chen, T. Yi, F. Li and C. Huang, J. Mater. Chem., 2008, 18, 886; (b) A. Das and S. Ghosh, Angew. Chem., Int. Ed., 2014, 53, 2038; (c) M. A. Castilla, B. Dietrich and J. D. Adams, Gels, 2018, 4.

7 (a) M. Irie, T. Fukaminato, K. Matsuda and S. Kobatake, Chem. Rev., 2014, 114, 12174; (b) S.-Z. Pu, Q. Sun, C.-B. Fan, R.-J. Wang and G. Liu, J. Mater. Chem. C, 2016, 4, 3075; (c) T. Mosciatti, M. G. del Rosso, M. Herder, J. Frisch, N. Koch, S. Hecht, E. Orgiu and P. Samorì, Adv. Mater., 2016, 28, 6606.

8 (a) M. Morimoto, S. Kobatake and M. Irie, Adv. Mater., 2002, 14, 1027; (b) M. Morimoto, S. Kobatake and M. Irie, J. Am. Chem. Soc., 2003, 125, 11080; (c) K. Higashiguchi, K. Matsuda, N. Tanifuji and M. Irie, J. Am. Chem. Soc., 2005, 127, 8922; (d) T. J. Wigglesworth and N. R. Branda, Chem. Mater., 2005, 17, 5473.

9 A. J. Myles and N. R. Branda, Adv. Funct. Mater., 2002, 12, 167.

10 T. B. Norsten and N. R. Branda, J. Am. Chem. Soc., 2001, 123, 1784. 11 (a) S. Ishida, T. Fukaminato, D. Kitagawa, S. Kobatake, S. Kim, T. Ogata and S. Kurihara, Chem. Commun., 2017, 53, 8268; (b) J. Chen, P. Zhang, G. Fang, P. Yi, F. Zeng and S. Wu, J. Phys. Chem. B, 2012, 116, 4354; (c) L. Zhu, W. Wu, M.-Q. Zhu, J. J. Han, J. K. Hurst and A. D. Q. Li, J. Am. Chem. Soc., 2007, 129, 3524; (d) S. Ishida, T. Fukaminato, S. Kim, T. Ogata and S. Kurihara, Chem. Lett., 2017, 46, 1182; (e) K. Watanabe, H. Hayasaka, T. Miyashita, K. Ueda and K. Akagi, Adv. Funct. Mater., 2015, 25, 2794; $(f)$ S. Kim, S.-J. Yoon and S. Y. Park, J. Am. Chem. Soc., 2012, 134, 12091; $(g)$ J. Bu, K. Watanabe, H. Hayasaka and K. Akagi, Nat. Commun., 2014, 5, 3799.

12 C. Li, Y. Zhang, J. Hu, J. Cheng and S. Liu, Angew. Chem., Int. Ed., 2010, 49, 5120 .

13 (a) S. A. Díaz, L. Giordano, T. M. Jovin and E. A. Jares-Erijman, Nano Lett., 2012, 12, 3537; (b) S. A. Díaz, F. Gillanders, K. Susumu, E. Oh, I. L. Medintz and T. M. Jovin, Chem. - Eur. J., 2017, 23, 263.

14 (a) H.-J. Kim, D. R. Whang, J. Gierschner, C. H. Lee and S. Y. Park, Angew. Chem., Int. Ed., 2015, 54, 4330; (b) W. Tian, J. Zhang, J. Yu, J. Wu, J. Zhang, J. He and F. Wang, Adv. Funct. Mater., 2018, 28, 1703548.

15 (a) J. W. Chung, S.-J. Yoon, S.-J. Lim, B.-K. An and S. Y. Park, Angew. Chem., Int. Ed., 2009, 48, 7030; (b) A. Kishimura, T. Yamashita and T. Aida, J. Am. Chem. Soc., 2005, 127, 179; (c) D. Kim, J. E. Kwon and S. Y. Park, Adv. Funct. Mater., 2018, 28, 1706213; (d) Q. Chen, D. Zhang, G. Zhang, X. Yang, Y. Feng, Q. Fan and D. Zhu, Adv. Funct. Mater., 2010, 20, 3244.

16 (a) M. Bälter, S. Li, M. Morimoto, S. Tang, J. Hernando, G. Guirado, M. Irie, F. M. Raymo and J. Andréasson, Chem. Sci., 2016, 7, 5867; (b) G. Naren, C.-W. Hsu, S. Li, M. Morimoto, S. Tang, J. Hernando, G. Guirado, M. Irie, F. M. Raymo, H. Sundén and J. Andréasson, Nat. Commun., 2019, 10, 3996.

17 C.-W. Hsu, C. Sauvée, H. Sundén and J. Andréasson, Chem. Sci., $2018,9,8019$.

18 K. Uno, H. Niikura, M. Morimoto, Y. Ishibashi, H. Miyasaka and M. Irie, J. Am. Chem. Soc., 2011, 133, 13558.

19 (a) A. Axelsson, L. Ta and H. Sundén, Eur. J. Org. Chem., 2016, 3339; (b) L. Ta, A. Axelsson, J. Bijl, M. Haukka and H. Sundén, Chem. - Eur. $J ., 2014,20,13889$; (c) A. Axelsson, L. Ta and H. Sundén, Catalysts, 2015, 5, 2052; (d) C. Sauvée, A. Ström, M. Haukka and H. Sundén, Chem. - Eur. J., 2018, 24, 8071.

20 P. Terech, D. Pasquier, V. Bordas and C. Rossat, Langmuir, 2000, 16, 4485. 21 J. B. Birks, D. J. Dyson, I. H. Munro and H. Flowers Brian, Proc. $R$. Soc. London, Ser. A, 1963, 275, 575.

22 (a) J. Matsui, M. Mitsuishi and T. Miyashita, J. Phys. Chem. B, 2002, 106, 2468; (b) T. Yamazaki, N. Tamai and I. Yamazaki, Chem. Phys. Lett., 1986, 124, 326.

23 (a) J. Lee, H. Jung, H. Shin, J. Kim, D. Yokoyama, H. Nishimura, A. Wakamiya and J. Park, J. Mater. Chem. C, 2016, 4, 2784; (b) C. Wang, Z. Wang, D. Zhang and D. Zhu, Chem. Phys. Lett., 2006, 428, 130.

24 Fig. S10 (ESI $\dagger$ ) demonstrates that eight distinct CIE coordinates can be accomplished with three different stimuli. However, those CIE coordinates are very close to each other due to the inefficient of FRET. 